\title{
The Complexity of Grid Coverage by Swarm Robotics
}

\author{
Yaniv Altshuler ${ }^{1,2}$ and Alfred M. Bruckstein ${ }^{1}$ \\ 1 Computer Science Department, Technion, Haifa, Israel \\ \{yanival, freddy\}@cs.technion.ac.il \\ 2 Deutsche Telekom Labs, Ben Gurion University, Beer Sheva, Israel
}

\begin{abstract}
In this paper we discuss the task of efficiently using ant-like robotic agents for covering a connected region on the $\mathbf{Z}^{2}$ grid, whose shape and size are unknown in advance, and which expands at a given rate. This is done using myopic robots, with no ability to directly communicate with each other, where each robot is equipped with only $O(1)$ memory. We show that regardless of the algorithm used, and the robots' hardware and software specifications, the minimal number of robots required in order to enable such coverage is $\Omega(\sqrt{n})$ (where $n$ is the initial size of the connected region). In addition, we show that when the region expands at a sufficiently slow rate, a team of $\Theta(\sqrt{n})$ robots could cover it in at most $O\left(n^{2} \ln n\right)$ time. Regarding the coverage of nonexpanding regions in the grid, we improve the current best known result of $O\left(n^{2}\right)$ by demonstrating an algorithm of worse case completion time of $O\left(\frac{1}{k} n^{1.5}+n\right)$, and faster for shapes of perimeter length which is shorter than $O(n)$.
\end{abstract}

\section{Introduction}

In this paper we examine a problem in which a group of ant-like robotic agents must cover an unknown region in the grid, that possibly expands over time. This problem is also strongly related to the problem of distributed search after mobile and evading target(s) $[3,4]$ or the problems discussed under the names of "Lions and Men" pursuits [6]. We analyze such issues using the results presented in [1, 2,11], concerning the Cooperative Cleaners problem, a problem that assumes a regular grid of connected 'tiles', part of which are 'dirty', the 'dirty' tiles forming a connected region of the grid. On this dirty grid region several agents move, each having the ability to 'clean' the place it is located in. In the dynamic variant of this problem a deterministic evolution of the environment in assumed, simulating a spreading contamination (or spreading fire).

First, we discuss the collaborative coverage of static grids. We demonstrate that the best completion time known to date $\left(O\left(n^{2}\right)\right.$, achievable for example using the $L R T A *$ search algorithm) can be improved to guarantee grid coverage in $O\left(\frac{1}{k} n^{1.5}+n\right)$ time. Later, we discuss the coverage of expanding domains. We show that using any conceivable algorithm, and using as sophisticated and potent robotic agents as possible, the minimal number of robots below which 
covering such a region is impossible equals $\Omega(\sqrt{n})$. We then show that when the region expands sufficiently slow (specifically, every $O\left(\frac{c_{0}}{\gamma_{1}}\right)$ time steps, where $c_{0}$ is the circumference of the region and where $\gamma_{1}$ is a geometric property of the region, ranging between $O(1)$ and $O(\ln n)$ ), a group of $\Theta(\sqrt{n})$ robots can successfully cover the region. Furthermore, we demonstrate that in this case a cover time of $O\left(n^{2} \ln n\right)$ is guaranteed.

\section{Related Work}

A search for analytic results concerning the completion time of ant-robots covering an area in the grid revealed only a handful of works. The main result in this regard is that of [7], where a swarm of ant-like robots is used to repeatedly cover an unknown area, using a real time search method called node counting, using integer markers that are placed on the graph's nodes. The cover time of teams of ant robots that use node counting is shown in $[8,9]$ to be $t_{k}(n)=O\left(n^{\sqrt{n}}\right)$, when $t_{k}(n)$ denotes the cover time of a region of size $n$ using $k$ robots. Another algorithm to be mentioned in this scope is $L R T A^{*}$, whose multi-robotics variant is shown in [8] to guarantee cover time of undirected connected graphs in polynomial time. Specifically, on grids it guarantee coverage in $O\left(n^{2}\right)$ time.

Vertex-Ant-Walk, a variant of the node counting algorithm presented in [12], is shown to achieve a coverage time of $O\left(n \delta_{G}\right)$, where $\delta_{G}$ is the graph's diameter, implying a worse-case completion time of $O\left(n^{2}\right)$ in grids. This work is based on a previous work in which a cover time of $O\left(n^{2} \delta_{G}\right)$ was demonstrated [10].

An algorithm named Exploration as Graph Construction, providing a coverage of degree bounded graphs in $O\left(n^{2}\right)$ time, can be found in [5]. Here a group of limited ant robots explore an unknown graph using special "markers".

We next show that the problem of collaborative coverage in static grids can be completed in $O\left(\frac{1}{k} n^{1.5}+n\right)$ time and that collaborative coverage of dynamic grids can be achieved in $O\left(n^{2} \ln n\right)$.

\section{The Dynamic Cooperative Cleaners Problem}

Following is a short summary of the Cooperative Cleaners problem, as appears in [11] (static variant) and [1,2] (dynamic variant). Assuming a discrete time, let the undirected graph $G(V, E)$ denote a two dimensional integer grid $\mathbf{Z}^{2}$, whose vertices (or "tiles") have a binary property called 'contamination'. Let $\operatorname{cont}_{t}(v)$ state the contamination state of the tile $v$ at time $t$, taking the values "on" or "off". Let $F_{t}$ be the contaminated sub-graph of $G$ at time $t$, and let $F_{0}$ be simply connected. Let a group of $k$ robots that can move on the grid $G$ (moving from a tile to its neighbor in one time step) be placed at time $t_{0}$ on $F_{0}$, at point $p_{0} \in F_{t}$. Each robot is equipped with a sensor capable of telling the status of all tiles in the digital sphere of diameter 7, which surrounds the robot. Each robot is equipped with a memory of size $O(1)$ bits. When a robot moves to a tile $v$, it has the possibility of cleaning this tile (i.e. causing $\operatorname{cont}(v)$ to become off. The robots do not have any prior knowledge of the shape or size of the sub-graph $F_{0}$.Every 
$d$ time steps, the contamination spreads. That is, if $t=n d$ for some positive integer $n$, then $\forall v \in F_{t} \forall u \in 4-N$ eighbors $(v), \operatorname{cont}_{t+1}(u)=$ on. The robots' goal is to clean $G$ by eliminating the contamination entirely. It is important to note that no central control is allowed, no communication is allowed, and that the system is fully decentralized.

A Survey of Previous Results. The cooperative cleaners problem was previously studied in $[1,2,11]$. A cleaning algorithm called SWEEP was proposed (used by a decentralized group of simple mobile robots, for exploring and cleaning an unknown "contaminated" sub-grid $F$, expanding every $d$ time steps) and its performance analyzed. The SWEEP algorithm is based in a constant traversal of the contaminated region, preserving the connectivity of the region while cleaning all non critical points - points which when cleaned disconnect the contaminated region. Following are several results that we later use. Note that cleaning a region is equivalent to covering it, as the number of uncovered tiles is upper bounded by the number of remaining contaminated ones.

Result 1 (Cleaning a Non-expanding Contamination) The time it takes for a group of $K$ robots using the $\boldsymbol{S} \boldsymbol{W E E P}$ algorithm to clean a region $F$ of the grid is at most:

$$
t_{\text {static }} \triangleq \frac{8\left(\left|\partial F_{0}\right|-1\right) \cdot\left(W\left(F_{0}\right)+k\right)}{k}+2 k
$$

$W(F)$ denotes the depth of the region $F$ (the shortest path from some internal point in $F$ to its boundary, for the internal point whose shortest path is the longest) and $\partial F$ denotes the boundary of $F$, defined via $\partial F=\{v \mid v \in F \wedge 8-$ Neighbors $(v) \cap(G \backslash F) \neq \emptyset\}$.

Result 2 (Universal Lower Bound on Contaminated Area) Using any cleaning algorithm, the area at time $t$ of a contaminated region that expands every d time steps can be recursively lower bounded, as follows :

$$
S_{t+d} \geq S_{t}-d \cdot k+\left\lfloor 2 \sqrt{2 \cdot\left(S_{t}-d \cdot k\right)-1}\right\rfloor
$$

Here $S_{t}$ denotes the area of the contaminated region at time $t$ (such that $S_{0}=n$ ).

Result 3 (Upper Bound on Cleaning Time for $S$ WEEP on Expanding Domains) For a group of $k$ robot using the $\boldsymbol{S} \boldsymbol{W E E P}$ algorithm to clean a region $F$ on the grid, that expands every $d$ time steps, the time it takes the robots to clean $F$ is at most $d$ multiplied by the minimal positive value of the following two numbers :

$$
\frac{\left(A_{4}-A_{1} A_{3}\right) \pm \sqrt{\left(A_{1} A_{3}-A_{4}\right)^{2}-4 A_{3}\left(A_{2}-A_{1}-A_{1} A_{4}\right)}}{2 A_{3}}
$$

where:

$A_{1}=\frac{c_{0}+2-\gamma_{2}}{4}, A_{2}=\frac{c_{0}+2+\gamma_{2}}{4}, A_{3}=\frac{8 \cdot \gamma_{2}}{d \cdot k} \quad, \quad A_{4}=\gamma_{1}-\frac{\gamma_{2} \cdot \gamma}{d}$, 


$$
\begin{gathered}
\gamma_{1}=\psi\left(1+A_{2}\right)-\psi\left(1+A_{1}\right) \quad, \quad \gamma_{2}=\sqrt{\left(c_{0}+2\right)^{2}-8 S_{0}+8}, \\
\gamma=\frac{8\left(k+W\left(F_{0}\right)\right)}{k}-\frac{d-2 k}{\left|\partial F_{0}\right|-1}
\end{gathered}
$$

Here $c_{0}$ is the circumference of the initial region $F_{0}$, and where $\psi(x)$ is the Digamma function - the logarithmic derivative of the Gamma function.

\section{Grid Coverage - Analysis}

We first present the cover time of a group of robots operating in non-expanding domains, using the SWEEP algorithm.

Theorem 1. Given a connected region of $S_{0}=n$ tiles and perimeter $c_{0}$, then $k$ ant-like robots can cover it using $O\left(\frac{1}{k} S_{0}^{1.5}+S_{0}\right)$ time.

Proof. Since $\left|\partial F_{0}\right|=\Theta\left(c_{0}\right)$, and $W\left(F_{0}\right)=O\left(\sqrt{S_{0}}\right)$, recalling Result 1 we see that:

$$
t_{k}(n)=t_{\text {static }}(k)=O\left(\frac{1}{k} \sqrt{S_{0}} \cdot c_{0}+c_{0}+k\right)
$$

As $c_{0}=O\left(S_{0}\right)$ and as for practical reasons we assume that $k<n$ this equals :

$$
t_{k}(n)=t_{\text {static }}(k)=O\left(\frac{1}{k} S_{0}^{1.5}+S_{0}\right)
$$

We now examine the problem of covering expanding domains. The lower bound for the number of robots required for completing is as follows.

Theorem 2. Given a region of size $S_{0} \geq 3$ tiles, expanding every d time steps, then a team of less than $\frac{\sqrt{S_{0}}}{d}$ robots cannot clean the region, regardless of the algorithm used.

Proof. Recalling Result 2, and by assigning $k=\frac{\sqrt{S_{0}}}{d}$ we can see that :

$$
\Delta S_{t}=S_{t+d}-S_{t} \geq\left\lfloor 2 \sqrt{2 \cdot\left(S_{t}-\sqrt{S_{0}}\right)-1}\right\rfloor-\sqrt{S_{0}}
$$

For any $S_{0} \geq 3$, we see that $\Delta S_{0}>0$. In addition, for every $S_{0} \geq 3$ we can see that $\frac{d S_{t}}{d t}>0$ for every $t \geq 0$. Therefore, for every $S_{0} \geq 3$ the size of the region will be forever growing.

Corollary 1. Given a region of size $S_{0}$ tiles, expanding every d time steps, where $d=O(1)$ w.r.t $S_{0}$, then a team of less than $\Omega\left(\sqrt{S_{0}}\right)$ robots cannot clean the region, regardless of the algorithm used.

Theorem 3. Let $F$ be a region of size $S_{0}$ tiles, expanding every d time steps. $A$ team of $k$ robots located at $t=0$ on the same tile cannot clean $F$, regardless of the algorithm used, if $d^{2} k<\Omega(R(F))$, where $R(F)$ is the perimeter of the bounding rectangle of $F$. 
Proof. For every $v \in F$ let $l(v)$ denote the maximal distance between $v$ and any of the tiles of $F$, namely:

$$
l(v)=\max \{d(v, u) \mid u \in F\}
$$

Let $C(F)=l\left(v_{c}\right)$ such that $v_{c} \in F$ is the tile with minimal value of $l(v)$.

Let $v_{s}$ denote the tile the agents are located in at $t=0$. Let $v_{d} \in F$ denote some contaminated tile such that $d\left(v_{s}, v_{d}\right)=l\left(v_{s}\right)$. Regardless of the algorithm used by the agents, until some agent reaches $v_{d}$ there will pass at least $l\left(v_{s}\right)$ time steps. Let us assume w.l.o.g that $v_{d}$ is located to the right (or of the same horizontal coordinate) and to the top (or of the same vertical coordinate) of $v_{s}$. Then by the time some agent is able to reach $v_{d}$ there exists an upper-right quarter of a digital sphere of radius $\left\lfloor\frac{l\left(v_{s}\right)}{d}\right\rfloor+1$, whose center is $v_{d}$. The number of tiles in such a quarter of digital sphere equals :

$$
\frac{1}{2}\left\lfloor\frac{l\left(v_{s}\right)}{d}\right\rfloor^{2}+\frac{3}{2}\left\lfloor\frac{l\left(v_{s}\right)}{d}\right\rfloor+1=\Theta\left(\frac{l\left(v_{s}\right)^{2}}{d^{2}}\right)
$$

It is obvious that the region cannot be cleaned until $v_{d}$ is cleaned. Let $t_{d}$ denote the time at which the first agent reaches $v_{d}$. It is easy to see that $t_{d} \geq l\left(v_{s}\right)$. Therefore, at time $t_{d}$ there are $k$ agents that has to clean a region of at least $\Theta\left(\frac{l\left(v_{s}\right)^{2}}{d^{2}}\right)$ tiles, spreading every $d$ time steps. Using Theorem 2 we know that $k$ agents cannot clean an expanding region of $k=\frac{\sqrt{S_{0}}}{d}$ tiles. Namely, at time $t_{d}$ the $k$ agents could not clean the contaminated tiles if :

$$
d^{2} k<\Omega\left(l\left(v_{s}\right)\right)
$$

As $l\left(v_{s}\right) \geq C(F)$ we know that $k$ agents could not clean an expanding contaminated region where : $d^{2} k<\Omega(C(F))$. It is easy to see that for every region $F$, if $R(F)$ is the length of the perimeter of the bounding rectangle of $F$ then $C(F)=\Theta(R(F))$.

Lemma 1. For every connected region of size $S_{0} \geq 3$ and perimeter of length $c_{0}$ :

$$
\frac{1}{2} c_{0}<\gamma_{2}<c_{0}
$$

Proof. let us assume by contradiction that $\left(c_{0}+2\right)^{2} \leq\left(8 S_{0}+8\right)$, implying $c_{0} \leq$ $\sqrt{8 S_{0}+8}-2$. However, the minimal circumference of a region of size $S_{0}$ is achieved when the region is arranged as an 8-connected digital sphere, in which $c_{0} \geq 4 \sqrt{S_{0}}-4$, contradicting the assumption that $c_{0} \leq \sqrt{8 S_{0}+8}-2$ for $S_{0}>5$ and hence, $\gamma_{2} \in \mathbb{R}$.

Let us assume by contradiction that $\gamma_{2}<\frac{1}{2} c_{0}$. This in turn implies :

$$
c_{0}<-\frac{16}{6}+\sqrt{10 \frac{2}{3} S_{0}-8 \frac{8}{9}}<3.266 \sqrt{S_{0}}-2
$$


We know that $c_{0} \geq 4 \sqrt{S_{0}}-4$, which contradicts the assumption that $\gamma_{2}<\frac{1}{2} c_{0}$ for every $S_{0} \geq 3$. Let us assume by contradiction that $\gamma_{2}>c_{0}$, implying that $c_{0}>4 S_{0}-6$.

We know that $c_{0} \leq 2 S_{0}-2$ (as $c_{0}$ is maximized when the tiles are arranged in the form of a straight line), contradicting the assumption that $\gamma_{2}>c_{0}$ for every $S_{0} \geq 3$.

Lemma 2. For every connected region of size $S_{0} \geq 3$ and perimeter of length $c_{0}$ :

$$
\Omega(1)<\gamma_{1}<O(\ln n)
$$

Proof. Let us observe $\gamma_{1}: \gamma_{1} \triangleq \psi\left(1+\frac{c_{0}+2+\gamma_{2}}{4}\right)-\psi\left(1+\frac{c_{0}+2-\gamma_{2}}{4}\right)$. From Lemma 1 we can see that $1<\left(1+\frac{c_{0}+2-\gamma_{2}}{4}\right)<\frac{1}{4} c_{0}$. Note that $\psi(1)=-\hat{\gamma}$ where $\hat{\gamma}$ is the Euler-Mascheroni constant which equals approximately 0.57721 . In addition, $\psi(x)$ is monotonically increasing for every $x>0$. As $\psi(x)$ is upper bounded by $O(\ln x)$ for large values of $x$, we see that:

$$
-0.58<\psi\left(1+\frac{c_{0}+2-\gamma_{2}}{4}\right)<O(\ln n)
$$

From Lemma 1 we also see that $1<\left(1+\frac{c_{0}+2+\gamma_{2}}{4}\right)<\frac{1.5}{4} c_{0}$ meaning that :

$$
\psi\left(1+\frac{c_{0}+2+\gamma_{2}}{4}\right)=\Theta(\ln n)
$$

Combining equations 1 and 2 the rest is implied.

Theorem 4. Result 3 returns a positive real number for the covering time of a region of $S_{0}$ tiles that expands every d time steps, when the number of robots equals $\Theta\left(\sqrt{S_{0}}\right)$ and $d=\Omega\left(\frac{c_{0}}{\gamma_{1}}\right)$, where $\gamma_{1}$ defined in Result 3 shifts from $O(1)$ to $O\left(\ln S_{0}\right)$ as $c_{0}$ grows from $O\left(\sqrt{S_{0}}\right)$ to $O\left(S_{0}\right)$.

Proof. In order for Result 3 to yield a real number all the following must hold :

$$
\begin{gathered}
d \cdot k \neq 0 \quad, \quad|\partial F|>1 \quad, \quad A_{3} \neq 0 \quad, \quad\left(c_{0}+2\right)^{2}>8 S_{0}-8 \\
\left(A_{1} A_{3}-A_{4}\right)^{2} \geq 4 A_{3}\left(A_{2}-A_{1}-A_{1} A_{4}\right)
\end{gathered}
$$

The first and second requirements hold for every non trivial scenario. The third requirement is implied by the fourth. The fourth assumption is a direct result of Lemma 1.

As for the last requirement, we ask that $A_{1}^{2} A_{3}^{2}+A_{4}^{2} \geq 4 A_{2} A_{3}-4 A_{1} A_{3}-$ $2 A_{1} A_{3} A_{4}$, which subsequently means that we must have :

$$
\frac{\gamma_{2}^{2}}{d^{2} k^{2}}\left(c_{0}^{2}+\gamma_{2}^{2}-c_{0} \gamma_{2}\right)+\gamma_{1}^{2}+\frac{\gamma_{2}^{2} \gamma^{2}}{d}-\frac{\gamma \gamma_{1} \gamma_{2}}{d} \geq \frac{\gamma_{2}}{d k} \cdot O\left(\begin{array}{c}
\gamma_{2}-c_{0} \gamma_{1}+c_{0} \frac{\gamma_{2} \gamma}{d} \\
-\gamma_{1}+\gamma_{1} \gamma_{2}-\frac{\gamma_{2}^{2} \gamma}{d}
\end{array}\right)
$$

Using Lemma 1 and Lemma 2 we should make sure that : 


$$
\frac{\gamma_{2}^{2}}{d k^{2}} c_{0}^{2}+\gamma_{1}^{2} d+\gamma_{2}^{2} \gamma^{2}-\gamma \gamma_{1} \gamma_{2} \geq O\left(\frac{c_{0} \gamma_{2} \gamma_{1}}{k}+\frac{c_{0} \gamma_{2}^{2} \gamma}{d k}\right)
$$

Using $W(F)=O\left(\sqrt{S_{0}}\right)$ and $\Omega\left(\sqrt{S_{0}}\right)=|\partial F|=O\left(S_{0}\right)$ and dividing by $\gamma_{2}^{2}$ (which we know to be larger than zero), we can write the above as follows:

$$
\frac{c_{0}^{2}}{d k^{2}}+\frac{k^{2}+d \ln ^{2} S_{0}}{c_{0}^{2}}+1 \geq O\left(\frac{\ln S_{0}}{c_{0}}+\frac{k \ln S_{0}}{c_{0}^{2}}+\frac{\ln S_{0}}{k}+\frac{c_{0} \sqrt{S_{0}}}{d k^{2}}+\frac{c_{0}}{d k}+\frac{1}{d}\right)
$$

As $c_{0} \geq \sqrt{S_{0}}$ then $\frac{c_{0}^{2}}{d k^{2}} \geq \frac{c_{0} \sqrt{S_{0}}}{d k^{2}}$. In addition, $1 \geq \frac{1}{d}$ and also $1 \geq \frac{\ln S_{0}}{c_{0}}$ and $1 \geq \frac{\ln S_{0}}{k}$. In order to have also $1 \geq \frac{c_{0}}{d k}$ we must have $: d \cdot k=\Omega\left(c_{0}\right)$

In addition, we also require that the cleaning time $\mu$ is positive :

$$
A_{4}+\sqrt{\left(A_{1} A_{3}-A_{4}\right)^{2}-4 A_{3}\left(A_{2}-A_{1}-A_{1} A_{4}\right)}>A_{1} A_{3}
$$

For this to hold we shall merely require that $A_{2}-A_{1}-A_{1} A_{4} \leq 0$ (as $A_{3}$ is known to be positive). Assigning the values of $A_{1}, A_{2}, A_{4}$, this translates to $c_{0}+c_{0}^{2} \frac{\gamma}{d} \leq O\left(c_{0} \gamma_{1}\right)$.

Dividing by $c_{0}$ we can now write $c_{0}+\frac{c_{0} \sqrt{S_{0}}}{k}+k \leq d O\left(\gamma_{1}\right)$. As $c_{0}$ is the dominant element, we see that $d=\Omega\left(\frac{c_{0}}{\gamma_{1}}\right)$. which subsequently implies that : $\Omega\left(\sqrt{S_{0}}\right) \leq k \leq O\left(c_{0}\right)$. Therefore, we select the value of $k$ such that $k=\Theta\left(\sqrt{S_{0}}\right)$.

Theorem 5. The time it takes a group of $k=\Theta\left(\sqrt{S_{0}}\right)$ robots using the $\boldsymbol{S} \boldsymbol{W E E P}$ algorithm to cover a connected region of size $S_{0}$ tiles, that expands every $d=$ $\Omega\left(\frac{c_{0}}{\gamma_{1}}\right)$ time steps (where $\gamma_{1}$ is defined in Result 3), is upper bounded by $O\left(S_{0}^{2} \ln S_{0}\right)$. Proof. Recalling Result 1, as we want at least a single contamination spread we assume $\frac{8\left(\left|\partial F_{0}\right|-1\right) \cdot\left(W\left(F_{0}\right)+k\right)}{k}+2 k \geq d$. Observing Result 3 we now see that :

$$
\begin{gathered}
t_{\text {SUCCESS }}=d \cdot O\left(A_{1}+\frac{\left|A_{4}\right|}{A_{3}}+\sqrt{A_{1}^{2}+\frac{\left|A_{1} A_{4}\right|+A_{1}+A_{2}}{A_{3}}+\frac{A_{4}^{2}}{A_{3}^{2}}}\right) \leq \\
d \cdot O\left(c_{0}+\gamma_{2}+d k \frac{\gamma_{1}}{\gamma_{2}}+k \gamma+\sqrt{k} \frac{\sqrt{c_{0}+\gamma_{2}} \sqrt{d \gamma_{1}+\gamma_{2} \cdot \gamma}}{\sqrt{\gamma_{2}}}+\sqrt{\frac{k d}{\gamma_{2}}} \sqrt{c_{0}+\gamma_{2}}\right)
\end{gathered}
$$

Using the fact that $\gamma_{2}=\Theta\left(c_{0}\right)$ (Lemma 1) we can rewrite this expression as :

$$
d \cdot O\left(c_{0}+d k \frac{\gamma_{1}}{c_{0}}+k \gamma+\sqrt{k} \sqrt{d \gamma_{1}+c_{0} \gamma}+\sqrt{k d}\right)
$$

As $W\left(F_{0}\right)=O\left(\sqrt{S_{0}}\right)$, we can now upper bound $d$ as follows $: d=O\left(\frac{\sqrt{S_{0}} \cdot c_{0}}{k}+\right.$ $\left.c_{0}+k\right)$. Therefore, $|\gamma|$ can now be written as $|\gamma|=O\left(\frac{\sqrt{S_{0}}}{k}+\sqrt{S_{0}}+\frac{k}{\sqrt{S_{0}}}+1\right)$. Remembering that $O\left(\sqrt{S_{0}}\right) \leq c_{0} \leq O\left(S_{0}\right)$ we can rewrite Equation 3 as follows : $d \cdot O\left(c_{0}+d k \frac{\gamma_{1}}{c_{0}}+k \sqrt{S_{0}}+\frac{k^{2}}{\sqrt{S_{0}}}+\sqrt{k c_{0}}\left(\sqrt{\gamma_{1}}+\sqrt[4]{S_{0}} \sqrt{\frac{\gamma_{1}}{k}}+\sqrt[4]{S_{0}}+\frac{\sqrt{k}}{\sqrt[4]{S_{0}}}\right)+\sqrt{k d}\right)$ which 2 can simplify to $d \cdot O\left(c_{0}+k \sqrt{S_{0}} \ln S_{0}+\frac{k^{2}}{\sqrt{S_{0}}}+\sqrt{c_{0} \ln S_{0} \sqrt{S_{0}}}+\sqrt{c_{0} k \sqrt{S_{0}}}\right)$.

Assuming that $k>O\left(\ln S_{0}\right)$ and as $c_{0}=O\left(S_{0}\right)$ we can now write :

$O\left(\frac{S_{0}^{2.5}}{k}+S_{0}^{2} \ln S_{0}+S_{0}^{1.5} k \ln S_{0}+k^{2} \sqrt{S_{0}} \ln S_{0}+\frac{k^{3}}{\sqrt{S_{0}}}+\frac{S_{0}^{2.25}}{\sqrt{k}}+S_{0}^{1.75} \sqrt{k}+S_{0}^{0.75} k^{1.5}\right)$ 


\section{Conclusions}

In this paper we have discussed the covering of a connected region on the grid using collaborate ant-like robotics system. We have shown that for static regions this can be done in $O\left(\frac{1}{k} \sqrt{n} \cdot c_{0}+c_{0}+k\right)$ time, equals $O\left(\frac{1}{k} n^{1.5}+n\right)$ time in the worst case. In addition, we have shown that when a region expands in a constant rate, a team of $\Theta(\sqrt{n})$ robots can still be guaranteed to clean or cover it, in $O\left(n^{2} \ln n\right)$ time.

In addition, we have shown that teams of less than $\Omega(\sqrt{n})$ robots can never cover a region that expands every $O(1)$ time steps, regardless of their sensing capabilities, communications and memory resources, or algorithm used. As to regions that expand slower, two impossibility results were shown. First, a region of $n$ tiles that expands every $d$ time steps cannot be covered by a group of $k$ agents if $d k \leq O(\sqrt{n})$. Theorem 4 guarantees a coverage when $d k=\Omega\left(\frac{n^{1.5}}{\ln n}\right)$, or even for $d k=\Omega(n)$ ) (when the region's perimeter $c_{0}$ equals $O(n)$ ).

Second, a spreading region cannot be covered when $d^{2} k$ is smaller than the order of the perimeter of the bounding rectangle of the region (which is $O(n)$ in the worse case and $O(\sqrt{n})$ for "round shapes"). Theorem 4 guarantees a coverage when $d^{2} k=\Omega\left(\frac{n^{2.5}}{\ln ^{2} n}\right)$, or for $d^{2} k=\Omega\left(n^{1.5}\right)\left(\right.$ when $\left.c_{0}=O(n)\right)$.

\section{References}

1. Altshuler, Y., Bruckstein, A., Wagner, I.: Swarm robotics for a dynamic cleaning problem. In: IEEE Swarm Intelligence Symposium. pp. 209-216 (2005)

2. Altshuler, Y., Wagner, I., Yanovski, V., Bruckstein, A.: Multi-agent cooperative cleaning of expanding domains. International Journal of Robotics Research (2010)

3. Altshuler, Y., Yanovsky, V., Bruckstein, A., Wagner, I.: Efficient cooperative search of smart targets using uav swarms. ROBOTICA 26, 551-557 (2008)

4. Borie, R., Tovey, C., Koenig, S.: Algorithms and complexity results for pursuitevasion problems. The Intl. Joint Conf. on AI (IJCAI) pp. 59-66 (2009)

5. Dudek, G., Jenkin, M., Milios, E., Wilkes, D.: Robotic exploration as graph construction. IEEE Transactions on Robotics and Automation 7, 859-865 (1991)

6. Isler, V., Kannan, S., Khanna, S.: Randomized pursuit-evasion with local visibility. SIAM Journal of Discrete Mathematics 20, 26-41 (2006)

7. Koenig, S., Liu, Y.: Terrain coverage with ant robots: A simulation study. In: AGENTS'01

8. Koenig, S., Szymanski, B., Liu, Y.: Efficient and inefficient ant coverage methods. Annals of Mathematics and Artificial Intelligence 31, 41-76 (2001)

9. Szymanski, B., Koenig, S.: The complexity of node counting on undirected graphs. Technical Report, CS Department, Rensselaer Polytechnic Institute (1998)

10. Thrun., S.B.: Efficient exploration in reinforcement learning - technical report cmu-cs-92-102. Technical report, Carnegie Mellon University (1992)

11. Wagner, I., Altshuler, Y., Yanovski, V., Bruckstein, A.: Cooperative cleaners: A study in ant robotics. The Intl. J. of Robotics Res. (IJRR) 27(1), 127-151 (2008)

12. Wagner, I., Lindenbaum, M., Bruckstein, A.: Efficiently searching a graph by a smell-oriented vertex process. Annals of Math. and AI 24, 211-223 (1998) 